\title{
Constellation Multi-Objective Optimization Design Based on QoS and Network Stability in LEO Satellite Broadband Networks
}

\author{
Dawei Yan ${ }^{1}$, Peng You ${ }^{1 *}$, Cong Liu ${ }^{2}$, Shaowei Yong ${ }^{1}$ and Dongfang Guan ${ }^{1}$ \\ ${ }^{1}$ Institute of Electronic Science and Engineering, National University of Defense Technology \\ Changsha, Hunan 410073, China \\ [e-mail: yandawei-1986@163.com, ypnudt@126.com, ysw_nudt@vip.126.com,gdfguandongfang@163.com] \\ ${ }^{2}$ College of Mathematics and Information Science, Baoji University of Arts and Sciences \\ Baoji, Shaanxi 721013, China \\ [e-mail: bjsjlc@163.com] \\ *Corresponding author: Peng You
}

Received August 17, 2017; revised February 15, 2018; revised July 10, 2018; accepted July 27, 2018;

published March 312019

\begin{abstract}
Low earth orbit (LEO) satellite broadband network is a crucial part of the space information network. LEO satellite constellation design is a top-level design, which plays a decisive role in the overall performance of the LEO satellite network. However, the existing works on constellation design mainly focus on the coverage criterion and rarely take network performance into the design process. In this article, we develop a unified framework for constellation optimization design in LEO satellite broadband networks. Several design criteria including network performance and coverage capability are combined into the design process. Firstly, the quality of service (QoS) metrics is presented to evaluate the performance of the LEO satellite broadband network. Also, we propose a network stability model for the rapid change of the satellite network topology. Besides, a mathematical model of constellation optimization design is formulated by considering the network cost-efficiency and stability. Then, an optimization algorithm based on non-dominated sorting genetic algorithm- II (NSGA- II) is provided for the problem of constellation design. Finally, the proposed method is further evaluated through numerical simulations. Simulation results validate the proposed method and show that it is an efficient and effective approach for solving the problem of constellation design in LEO satellite broadband networks.
\end{abstract}

Keywords: Constellation design, LEO satellite broadband network, quality of service (QoS) metrics, network stability factor, cost-efficient ratio, NSGA- II -IS

This work was supported in part by the National Natural Science Foundation of China under Grants 61601487. 


\section{Introduction}

\subsection{Background}

$\mathbf{W}_{\text {ith the development of astronautics science and technology, researchers take more }}$ attention to explore the space information network (SIN) [1]. SIN, consisting of satellites and satellite constellations at different Earth orbits for increasing the coverage area, can achieve real-time acquisition, transmission, and processing of spatial information with collaboration mechanisms [2]. As an important component in SIN, LEO satellite broadband network has unique capabilities in terms of low-propagation delay, wide area coverage, and Internet-based application to provide services anytime and anywhere. In order to achieve an LEO satellite broadband network, an appropriate satellite constellation is needed [3]. However, there are many challenges for constellation design to construct the LEO satellite network. For one thing, a complex issue arising out of constellation design is rooted in an unlimited choice of six parameters (altitude, eccentricity, inclination, argument of perigee, right ascension of the ascending node and mean anomaly) for each orbit [4]. Hence, constellation design problem is characterized by extremely high dimensionality. For another thing, different satellite constellations may present distinct topological dynamics under given continuously global coverage. The highly dynamic characteristics of constellations are an essential factor which affects the stability of satellite networks [5]. These are the main reasons for an extreme technology development delay in the research field of constellation design presently [6].

\subsection{Related Works}

Many researchers have done some investigations on constellation design. These topics mainly include constellation design on the basis of geometric analytical and by using multi-objective optimization methodology.

(1) Constellation Design Based on Geometric Analytical: Constellation design methods have been developed based on the impacts of satellite orbital parameters on coverage performance. Walker Constellation is the most mature and widely-used design method for continuous global coverage by using circular orbits [7]. There are many real-world applications based on Walk Constellation, such as GPS, GALILEO, GLONASS and so forth. Mortari et al. [8] proposed a general method for constellation design, which named Flower Constellation (FC). Each satellite in FC is placed in elliptical orbits. H. Keller et al. investigated the design method of near-polar orbital constellations and successfully used this method in the optimal deployment of Iridium system [9]. Yuri proposed a geometric approach for complex coverage constellations by two-dimensional maps of visibility properties [10]. All of these approaches can solve the complex problem by reducing the dimensionality of constellation design based on some specific assumption. However, the discovery and development of new and useful solutions are always prevented because of the assumptions. Therefore, the novel design approach should be explored to extend the feasible solution space, which could provide more excellent constellation for a satellite system.

(2) Constellation Design via Multi-Objective Optimization Methodology: With the rapid advancement of the evolutionary algorithm, multi-objective evolutionary algorithms have been applied to design optimal constellation schemes for the global or regional coverage satellite system. Williams et al. [11] used the multi-objective evolutionary algorithm to find constellations with minimal average and maximum revisit time for user terminals on the entire 
Earth. Mason et al. [12] proposed an optimization design method for satellite constellation with continuous global coverage by using a genetic algorithm. More recently, researches have applied state-of-art multi-objective evolutionary algorithms and parallel computing for lager constellation design in order to reduce runtime [13],[14]. Williams et al. [15] presented a method of constellation design from the perspective of the evolutionary search. Global coverage constellation can be achieved by getting rid of the assumption of rigidly symmetric orbits. However, these approaches are significantly influenced by the optimization algorithm, and what's more, coverage capability is considered only as its optimization objective.

Currently, SIN becomes increasingly important in both civilian and military fields, in which constellation design is needed not only coverage capability but also some new requirements [16],[17]. For constructing a global satellite communication system, constellation design is a critical preparation before launching the satellites. Jiang et al. [18] proposed to establish an asymmetric Equatorial-Polar plane constellation for global communication without inter-orbit inter-satellite links (ISLs). This constellation provides an excellent coverage performance with less satellite and only intra-orbit ISLs to reduce the system cost. Jilla et al. translated the distributed satellite system conceptual design problem into a multi-objective optimization problem by considering QoS metrics without establishing ISLs [19].

All of the above works focus on the methodology of constellation design, however, only a few of them concentrates on both the QoS and networking during solving this complex problem. It is still inadequate to systematic research of constellation design in LEO satellite broadband network. Therefore, this paper is an attempt to develop a multi-objective optimization methodology for the constellation design problem with considering both the QoS metrics and establishing ISLs.

\subsection{Contributions}

This paper is developed an optimization design methodology for LEO satellite constellation based on a constrained multi-objective evolutionary algorithm. The main contributions are listed in the following:

(1) Several design criteria including network performance and coverage capability are merged into the unified frame for optimization design of the satellite constellation. The optimization techniques will be used to generate sets of Pareto solutions that show the tradeoff between network stability and cost-efficiency.

(2) The QoS metrics linked to network performance are defined and formulated as the constraints for the optimization model of constellation design. The stability factor is proposed to evaluate the dynamic topology of the satellite constellation in the case of the relative motion of satellites.

(3) A specialized optimization algorithm is proposed to solve the problem of satellite constellation design. Results demonstrate the efficiency of proposed optimization methodology by some numerical simulations.

The rest of this article is organized as follows. Section 2 gives the LEO satellite broadband network model. In section 3, we formally define the QoS metrics and stability model for the satellite network. In section 4, the optimization model of constellation design is established. Section 5 introduces the constrained multi-objective evolutionary algorithm. The simulation results and an example analysis is shown in section 6 . Finally, section 7 concludes the paper by pointing out a few possible future directions. 


\section{LEO Satellite Broadband Network Model}

LEO satellite broadband network can be achieved via ISLs based on a constellation, in which users are confronted with many challenges, such as complex environment, scarce wireless resources and limited network capacity [21]. How to design an excellent constellation and user links to match the user requirements is the urgent problems to be solved.

\subsection{Constellation Model}

The global or latitudinal coverage can be realized by using Walker Constellations with symmetrical structure [7]. A Walker Constellation consists of three integer parameters: the number of satellites $T$, the number of orbit planes $P$, and the phase factor between satellites in adjacent planes $F$, and two other orbital parameters: the orbital height $h$, and the inclination $i$. For reducing the computational complexity, Walker Constellation is chosen as the constellation model of the LEO satellite broadband network.

(1) Network Coverage. The satellite coverage area is determined by the minimum elevation of the user and line-of-sight propagation. Coverage model of the circular orbit satellite is shown in Fig. 1. Assuming that the Earth is a regular sphere, a satellite coverage area $A$ is given by

$$
\left\{\begin{array}{c}
A=2 \pi R_{e}^{2}(1-\cos \varphi) \\
\varphi=\arccos \left[\frac{R_{e}}{R_{e}+h} \cos E\right]-E
\end{array}\right.
$$

where $\varphi$ is geocentric angle between satellite and user terminal, $R_{e}$ is the Earth's radius, $h$ is the orbit height of the satellite, and $E$ is the minimum elevation.

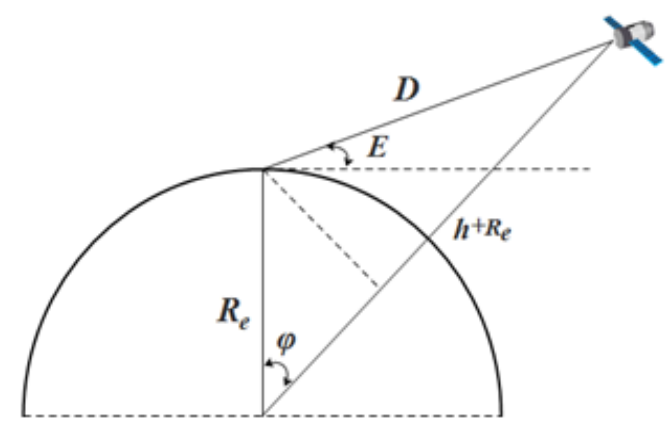

Fig. 1. Coverage model of circular orbit satellite

Equation (1) shows that the coverage area of a satellite with specified altitude is inversely proportional to the minimum elevation. This indicates that the larger the minimum elevation angle is, the smaller the satellite coverage area is. Meanwhile, the minimum elevation affects the performance of wireless communication links between users and satellite because the distance $(D)$ between users and satellite increases as the minimum elevation is decreased. Therefore, taking the complex surface of the Earth into consideration, the elevation larger than $10^{\circ}$ is necessary to sustain effective satellite communication [22].

Due to a satellite coverage area is limited, it is needed for a satellite constellation to expand the coverage area. For evaluating coverage performance of the constellation, this paper introduces Percentage of coverage $\eta_{\text {Coverage }}$ by numerical simulation as follows: 


$$
\eta_{\text {Coverage }}=\frac{100}{N_{\text {tol }}} \sum_{i=1}^{N_{\text {tol }}} L \operatorname{cov}_{i}
$$

where $L \operatorname{cov}_{i} \in\{0,1\}$ is evaluated whether the ith grid point is covered by a satellite. Set $L \operatorname{cov}_{i}$ to 1 If the ith grid point is covered by at least one satellite, 0 otherwise. $N_{\text {tol }}$ denotes the total number of grid point evenly distributed on the Earth surface.

(2) Network Connectivity. The frequent handover of ISLs is caused by dynamic topology and periodicity of the constellation. It not only increases the difficulty and cost of the constellation design but also directly affects the transmission performance of the network. In order to eliminate these impacts, the best way is to establish the permanent ISL between the satellites in the constellation. Fig. 2 shows the two-dimensional geometric relationship between two satellites in different orbits. The condition of permanent visibility between two satellites is that the $x^{\prime}$ coordinate of sat2 is always greater than $-d$ to avoid sat2 entering into sat1's invisible area, which is denoted by the mathematical formulae as follow [23]

$$
\frac{r}{R_{e q}} \geq \sqrt{1+\frac{\left(1-\varepsilon_{\min }\right)^{2}}{1-\varepsilon_{\min }^{2}}}
$$

where $r$ is the radius of the orbit, $R_{e q}$ denotes the equivalent Earth radius after considering the influence of atmospheric and topography, and $\varepsilon_{\min }$ denotes the minimum x'-axis coordinate of sat2.

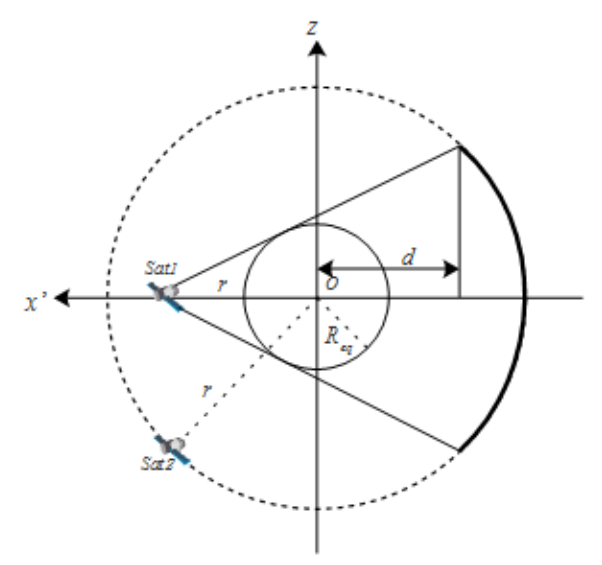

Fig. 2. Geometry relationship between two satellites in different orbits ${ }^{[23]}$

In order to achieve satellite network, establishing ISLs between the satellites in a constellation must obey the connection rule: there are four ISLs for each satellite in the constellation[24]. Intra-orbit ISL is a communication link between two neighboring satellites in the same orbit. For inter-orbit ISL, each satellite is connected to the two satellites in the left side and right side of adjacent orbits. The initial phase of two satellites connecting through inter-orbit ISL is the angle caused by a satellite in the backward orbit just over ascending node when the other one in the forward orbit is locating at the ascending node.

The connectivity of a LEO satellite broadband network can be defined as:

$$
\eta_{\text {NC }}=\frac{100}{N_{i s l} L} \sum_{i=1}^{N_{s}-1} \sum_{j=i+1}^{N_{s}} \sum_{k=1}^{L} \nu_{i s l}^{i, j, k}
$$


where $v_{i s l}^{i, j, k} \in\{0,1\}$ is evaluated whether there is a communucation link between the ith and $j t h$ satellites at the kth time instant. $N_{i s l}$ is the number of ISLs $\left(N_{i s l}=2 N_{s}\right.$ for a constellation of $N_{s}$ satellites). We consider the LEO satellite broadband network as an all-to-all network (e.g., $\left.\eta_{N C}=100 \%\right)$.

\subsection{Link Budget Mode}

Assuming that the LEO satellite broadband network adopts uncoded binary phase shift keying (BPSK) modulation scheme with coherent demodulation. Therefore, bit error rate (BER) is obtained by [25]

$$
p_{e}=\frac{1}{2}\left(1-e r f \sqrt{E_{b} / N_{0}}\right)
$$

Under given the BER of the satellite network, the corresponding signal-to-noise ratio (SNR) threshold $E_{b} / N_{0}$ can be calculated from equation (5).

The data rate $R$ of the downlink in the satellite network can be given by [25]:

$$
R=\frac{P_{S a t} G_{S a t} G_{r} L_{D} L_{R M}}{S N R k T}
$$

where $P_{\text {Sat }}$ is the transmission power of a satellite, $G_{\text {Sat }}$ is gain of the satellite transmitting antenna, $G_{r}$ is gain of the receiving terminal, $L_{R M}$ denotes link margin, $T$ denotes noise temperature of the receiving terminal, $k$ is the Boltzmann's constant, SNR is obtained from equation (5) under given BER, and $L_{D}$ denotes the downlink propagation loss. $L_{D}=L_{F D}+A_{R A I N}+L_{a}+L_{I}$, where $L_{F D}$ denotes free-space loss, $A_{\text {RAIN }}$ denotes rain fade in the troposphere, $L_{a}$ denotes the atmospheric absorption loss, and $L_{I}$ is all the interference losses. In order to ensure efficiency of the wireless link, a certain margin is needed to compensate satellite links.

To simplify the module, the characteristics of satellite antennas are described by user requirements without a particular shaped antenna (i.e. phased array antennas, parabolic antenna and so on). The satellite antenna as an abstract resource could "intelligent" dynamically configure beam patterns to the coverage area. Then the satellite antenna gain $G_{\text {sat }}$ is [26]

$$
G_{\text {sat }}=\eta_{\text {sat }} \frac{4 \pi A_{\text {sat }} f_{c}^{2}}{c^{2}}
$$

where $A_{\text {sat }}$ is the equivalent area of the satellite downlink antenna, $\eta_{\text {sat }}$ denotes the antenna efficiency, and $f_{c}$ is the downlink transmission frequency.

The satellite capacity $C_{\text {sat }}$ can be calculated depending on equation (6) and (7) as follows:

$$
C_{\text {sat }}=\frac{R \eta_{\text {MAE }}}{R_{\text {user }}}
$$

where $\eta_{\text {MAE }}$ denotes the efficiency of multi-access, $R_{\text {user }}$ is the date rate provided for user terminals (i.e.T1 link, a standard is to set the date rate of broadband communication at least 1.544 Mbps by the Federal Communication Commission ). It is worth noting that different 
information rate standards of broadband communication will not affect the constellation design and the numerical results are just different.

Therefore, the network capacity is defined as the total number of the user terminals servered by LEO satellite broadband network in all target grids . The maximum network capacity $C_{\text {Max }}$ is written as

$$
C_{\text {Max }}=\sum_{\text {grid }=1}^{N_{\text {grid }}} \min \left\{C_{\text {Sat }}(\text { grid }), C_{\text {demand }}(\text { grid })\right\}
$$

where $N_{\text {grid }}$ denotes the grid number on the Earth surface, $C_{\text {sat }}$ (grid) is the satellite capacity $C_{\text {sat }}$ in the target grid, and $C_{\text {demand }}$ ( grid) is total demand of the user terminals in the same grid. The minimum value between $C_{\text {sat }}($ grid $)$ and $C_{\text {demand }}($ grid $)$ is chosen as $C_{\text {Max }}($ grid $)$. Assuming that the same number of the user terminals are uniformly distributed in each grid.

\subsection{Assumptions}

As a general optimization design methodology of the satellite constellation, the specific communication scheme and antenna technology are not discussed in this article. Without loss of generality, the following assumptions are needed to make for simplicity.

(1) Due to the dynamic characteristics of the LEO satellite constellation, a handover between the satellite and user will be established frequently. Moreover, the distribution of the users and communication service in the coverage area is exceedingly uneven. It is assumed that the satellite network can provide QoS guarantee for users by resource management [27].

(2) The satellites are interconnected by ISLs. It is assumed that permanent ISL can be established between two satellites as long as equation (3) is met.

(3) The user uplink is ideal and the downlink capacity is only taken into account in constellation design.

(4) Due to the effect of J2 perturbation and gravity from other celestial bodies in space, satellite orbit will drift. Assuming that self-adaption control system is used for stationkeeping of a constellation.

These assumptions can be achieved with the help of advanced technology in aerospace engineering, electronic engineering, and other related technologies.

\section{QoS Metrics and Network Stability}

\subsection{QoS Metrics}

The QoS is derived from the contradictory between the quality and the quantity of services in the communication networks. But a unified definition on the QoS has not been formed. For International Telecommunication Union (ITU), the QoS is described as a set of service quality requirements based on the effect of the services for the users. In order to take full use of resources, administrators need to fully investigate characteristic of service requirements for allocating reasonably network resource. The QoS metrics are described as transmission delay, delay jitter, bandwidth, packet loss ratio and reliability and so on. From the perspective of the distributed satellite system, Jilla et al. defined the QoS metrics as four parameters (such as isolation, integrity, rate, and availability) to evaluate the performance of the network [19]. Dong et al. established QoS metrics which are appropriate to the requirement of HAP 
broadband network from the HAP constellation design perspective [28]. In this paper, we define the QoS metrics as the effectiveness and fairness of the mobile broadband services provided by the satellite network. The parameters of our proposed QoS metrics are listed in Table 1.

Table 1. The QoS metrics

\begin{tabular}{ccc}
\hline QoS Metrics & Equivalent Parameter & Symbol \\
\hline \multirow{2}{*}{ Effectiveness } & Signal-to-noise ratio & $S N R_{0}$ \\
& Date rate & $B E R_{0}$ \\
& Bit error rate & $R_{0}$ \\
\hline \multirow{2}{*}{ Fairness } & Percentage of coverage & $\eta_{\text {Coverage }}$ \\
& Network connectivity & $\eta_{N C}$ \\
\hline
\end{tabular}

The effectiveness of the services is mainly determined by three elements:

(1) Signal-to-noise ratio: which represents an approach of signal isolation for the LEO satellite broadband network. It indicates that whether the satellite network is able to identify the useful signals from the noise signals and different interference.

(2) Date rate: which measures the information transmission rate between source and destination nodes in the satellite network. In this paper, date rate represents that the network must provide the user terminals with the least information per second (bits/second).

(3) Bit error rate: Bit error rate (BER) is the number of bit error per unit time in digital transmission owing to noise, interference, or distortion. BER refers to the quality of the information being transmitted through the LEO satellite broadband network.

The fairness of the services characterizes the user requirements and the state of the network as follow:

(1) Coverage percentage: which is the ratio of the number of the grids covered by the satellites to the total number of the grids on the Earth surface. Percentage of coverage is quantified by a numerical simulation method in this paper.

(2) Network connectivity: which is used to describe the number of ISLs in the LEO satellite broadband network. It is obvious that the higher network connectivity means the better robustness of the network.

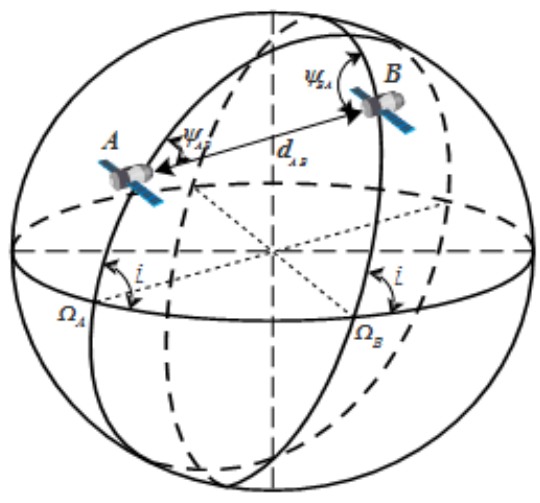

Fig. 3. Location of satellites on the celestial sphere 


\subsection{Satellite Network Stability Model}

In LEO satellite networks, due to the relative movements between satellites shown in Fig. 3, the network topology changes rapidly. Because of these rapid changes in topology, satellite networks will no longer be able to provide stable date flow interaction. And the factors affecting LEO satellite network stability can be outlined as two parts: the change rate of relative distance and azimuth angle of neighboring satellites [29],[30]. On the one hand, the relative distance variation can dramatically affect the reliability of information interaction between two satellites. The change rate of distance is too fast, and it is hard to establish an effective ISL. Meanwhile, the transmission attenuation will be more serious with the distance among satellite nodes increasing. On the other hand, when an antenna is used to track peer satellites with higher angular velocity, the excessive angular speed may have a significant impact on the stability of the satellites in orbit and therefore could deteriorate the stability and quality of ISLs. Moreover, satellite antennas also cannot afford such an excessive change rate of distance and azimuth angle to maintain the ISLs.

Therefore, it is necessary to design a reasonable satellite constellation for establishing ISLs in the case of the relative motion of satellites. To address this problem, ISLs should be well designed based on the distribution of satellites in a constellation. After reasonable design, it is available to build an efficient and reliable communication between the two satellites.

In this paper, our approach is to consider the factors affecting satellite network stability into the constellation optimization design process. Therefore, network stablity factor $\Psi$ is defined to characterize the stability of the LEO satellite network as follow

$$
\Psi=\alpha \dot{\Delta d}+\beta \dot{\Delta} \psi
$$

where $\dot{\Delta d}$ and $\Delta \dot{\psi}$ indicate distance rate and angular rate of the ISL, respectively. $\alpha$ and $\beta$ are the control parameter satisfying $\alpha+\beta=1$. They can be adjusted by considering the different focuses of establishing a ISL.

Distance rate of ISL [31] $\dot{\Delta d}$ is given by

$$
\dot{\Delta d}=E\left(\left|\frac{\partial \Delta d}{\partial t}\right|\right)
$$

where $\Delta d$ denotes the ISL's relative distance, $E(\bullet)$ is calculating mean value.

Similarly, angular rate of ISL[31] $\Delta \dot{\psi}$ is given by

$$
\Delta \dot{\psi}=E\left(\left|\frac{\partial \psi_{A B}}{\partial t}\right|\right)
$$

where $\psi_{A B}$ denotes the ISL's azimuth angle.

Under this definition, the smaller stability factor suggests a great opportunity to build a stable LEO satellite network. Then, we put the defined network stability factor into the constellation design objective functions. And the best stable satellite network can be found by minimizing the network stability factor. 


\section{Satellite Constellation Optimization Design}

To apply a multi-objective optimization tool for satellite constellation design, optimization variables, objective functions, and constraints should be determined on the basis of the performance requirements of LEO satellite broadband network.

\subsection{Optimization Variables}

Given a set of optimization variables, there will be a unique network architecture. For a small number of optimization variables, the size of the design space is decreased. Meanwhile, the number of optimization variables are less so that computational complexity will be greatly reduced.

The optimization variables, outlined in Table 2, contain seven parameters: the number of orbital planes, the number of satellites per orbital plane, phase factor, orbital height, inclination, the equivalent area of satellite downlink antenna, and transmission power of a satellite. The architecture of LEO satellite broadband network can be developed based on these key independent optimization variables. Note that a very small change in the optimization variables space could lead to a large jump in the design tradeoff space. Thus, to explore the optimal solutions is necessary by using an optimization algorithm.

Table 2. Optimization variables

\begin{tabular}{ccc}
\hline OptimizationVariable & Symbol & Uints \\
\hline Number of Orbital Planes & $P$ & - \\
Number of Satellite per Orbital Planes & $S$ & - \\
Phase Factor & $F$ & - \\
Orbit Height & $h$ & $\mathrm{~km}$ \\
Inclination & $i$ & $\mathrm{deg}$ \\
Equivalent Area of Downlink Antenna & $A_{\text {sat }}$ & $\mathrm{m}^{2}$ \\
Transmission power of the satellite & $P_{\text {Sat }}$ & $\mathrm{w}$ \\
\hline
\end{tabular}

\subsection{Optimization Model}

This paper is aimed to achieve a cost-efficient satellite network with high stability by optimization design of the satellite constellation. Therefore, a mathematical model for constellation optimization design is then formulated as

Objective:

Constraints:

Effectiveness

$\begin{array}{ll} & B E R \leq B E R_{0} \\ & R \geq R_{0} \\ \text { Fairness } & \eta_{\text {Coverage }}=100 \%\end{array}$




$$
\begin{gathered}
\eta_{N C}=100 \% \\
\text { Permanent Visibility } \frac{r}{R_{e q}} \geq \sqrt{1+\frac{\left(1-\varepsilon_{\min }\right)^{2}}{1-\varepsilon_{\text {min }}^{2}}}
\end{gathered}
$$

The first objective function is network capacity per cost (NCPC) $\Phi$. The NCPC is a function of the maximum capacity and potential cost of the satellite network. The satellite number $N_{\text {Sat }}$, equivalent area of the antenna $A_{\text {sat }}$ and transmission power $P_{\text {sat }}$ make up the potential cost of the network [28]. The second objective function is network stability factor (NSF) $\Psi$ defined as before. The optimization constraints include the QoS metrics $\left(S N R_{0}, B E R_{0}, R_{0}, \eta_{\text {DONC }}\right.$ and $\eta_{\text {Coverage }}$ ) and the condition of permanent visibility $r / R_{e q}$. Uper or lower bounds on the effectiveness of services are set as the first three constraints. And for the fairness of services, coverage percentage $\eta_{\text {Coverage }}$ and network connectivity $\eta_{N C}$ must equal to $100 \%$.

It should be noticed that the QoS metrics are transformed into boundary constraints rather than the optimization objectives in equation (13). Because this paper pays more attention to whether the QoS metrics of the whole network are satisfied and does not focus on how much redundant resource the satellite network can provide for a specific user.

\section{Optimization Algorithm}

From equation (13), the optimization model of constellation optimization design is characteristic of non-convex, nonlinear, and discontinuous. The specific optimization problem is difficult to be solved by the traditional optimization method.

\subsection{Integrating NSGA- II with Constraint Handling}

Recently, multi-objective evolutionary algorithms (MOEAs) have achieved great success in various fields with its low complexity and fast convergence. In particular, NSGA- II proposed by Deb has been increasingly used in various optimization fields [20]. However, the traditional EA cannot solve the optimization problems with constraints. Therefore, it is necessary to propose a new MOEA which can effectively handle the constraints.

The survey on constraint handling approaches for EA has been divided into four types: separation of objectives and constraints, decoders, penalty functions, and special operators [32]-[34]. The first one with the simple concept is often used to improve the reserved strategy of the solutions in NSGA- II [32]. The strategy of tournament selection or elitism can be improved as follow:

(1) The solution with the better value of objective function is selected between two feasible solutions.

(2) The feasible solution is chosen between a feasible and an infeasible solution.

(3) The solution with the lower constraint violations level is selected between two infeasible solutions.

Plenty of studies have proved that it is beneficial to reserve appropriate infeasible solutions in each generation. The selected infeasible solution is an especial link between the regions of feasible solutions. Such infeasible solutions are often better than some feasible ones and they should increase the opportunity to be reserved. Moreover, some feasible solutions approximating constraint values will be identified as infeasible solutions with high risks. 
According to the above analysis, this paper proposes an improved NSGA- II integrating with constraint handling method. This method is used to sort the infeasible solutions based on the constraint violation value. This new optimization algorithm is called NSGA- II based on Infeasible Sorting (NSGA- II -IS).

(1) Infeasible Sorting Operator: In each generation, the constraint violation values of all solutions are calculated on the basis of the constraints. The value of constraint violation reflects the degree of violating a constraint. The value 0 is allotted for a solution that does not violate a constraint. Otherwise, a non-zero value is allotted. A solution can get an ascending order by sorting the constraint violation value. In this sorted list, a solution with the least constraint violation value gets rank 1 and the solutions with the same constraint violation value get the same rank. All the constraints implement this sorting operator separately. Finally, constraint violation level of each solution is obtained by summing the ranks for each constraint which is violated.

(2) Modify Elitist Component: The parameter $\alpha$ is defined as the proportion of the infeasible solutions which are retained in the population. If the infeasible set is smaller than the $\alpha$-proportion, all the infeasible solutions are copied into the next generation. The other solutions of the new population are selected from the feasible set. If the feasible set includes fewer solution, all the feasible solutions are chosen and there will be filled new population with infeasible solutions.

(3) $\varepsilon$-Dominated Sorting [35]: The epsilon factor is used to control the overall archive size to ensure the diversity of evolution population. In each generation, the solutions of the population are divided into a feasible set and an infeasible set respectively. The feasible set is $\varepsilon$-dominated sorted of $k$ objectives. On the other hand, the infeasible set is $\varepsilon$-dominated sorted using $\mathrm{k}+1$ objectives (constraint violation level treat as the additional objective).

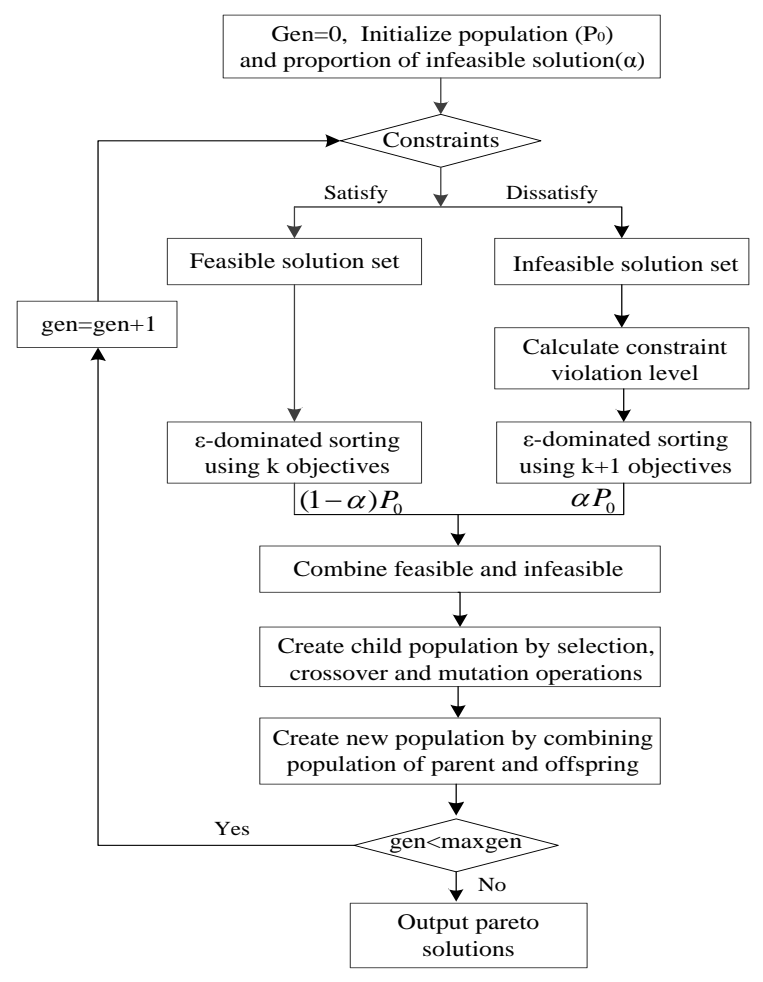

Fig. 4. The flow chart of NSGA- II -IS 
The flow chart of improved NSGA-II -IS based on infeasible sorting is shown in Fig. 4. Although this constraint handling approach is simpler, the advantages of this approach are as follows:

(1) A constraint problem is transformed into an unconstraint problem by taking the constraint violation level as an additional objective. The values of constraint violation in each infeasible solutions can be ignored.

(2) Infeasible solutions with higher ranks than some feasible solutions will extend the search space to the infeasible solutions area. It promotes the search of solutions towards the constraint boundary.

\subsection{Algorithm Testing}

In order to verify the effectiveness of the proposed algorithm, we use the CTP series of benchmark problems [36] as bi-objective optimization test problems. A population of size 200 and a maximum generation of 200 are used. The proportion of infeasible solutions remains at twenty percent $(\alpha=0.2)$. We have used the crossover parameter with $\eta \mathrm{c}=15$ and mutation parameter with $\eta \mathrm{m}=20$. A crossover probability of 0.9 and mutation probability of 0.1 are chosen. Fig. 5 shows the distributions of the 160 final feasible solutions for 2-objective test instance, CTP1, CTP3, CTP6, and CTP7. Simulation results show that the obtained solutions present good uniformity of diversity, and approach the true frontier of Pareto.

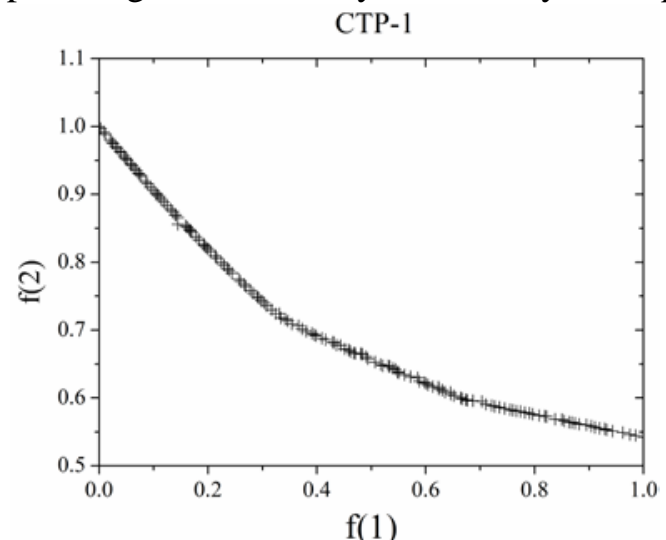

(a)

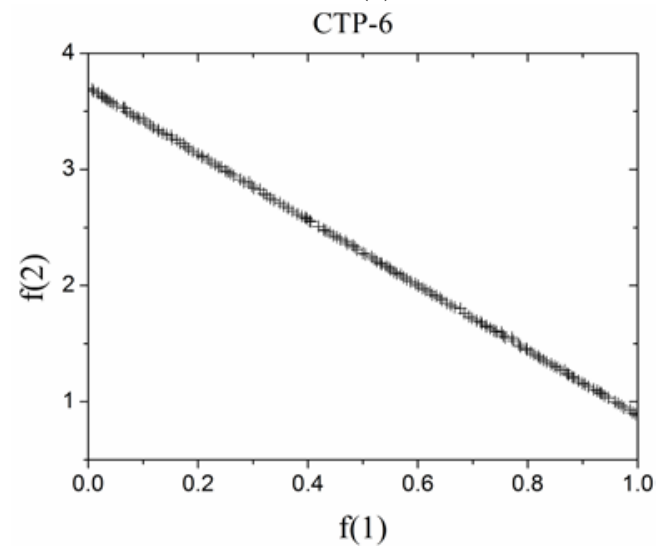

(c)

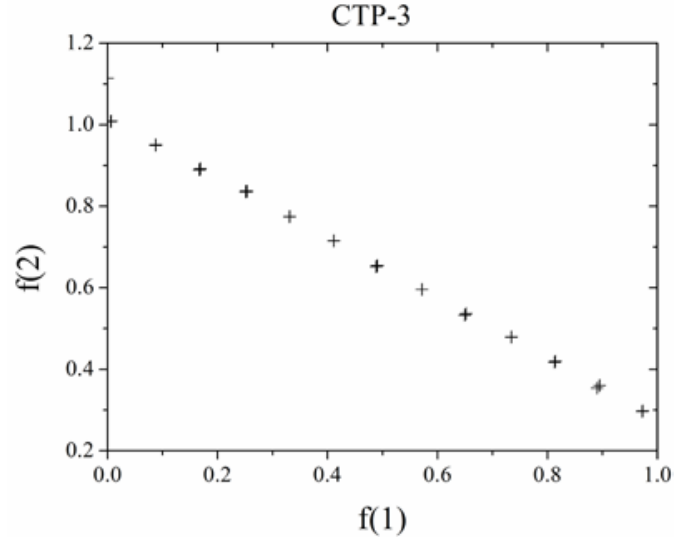

(b)

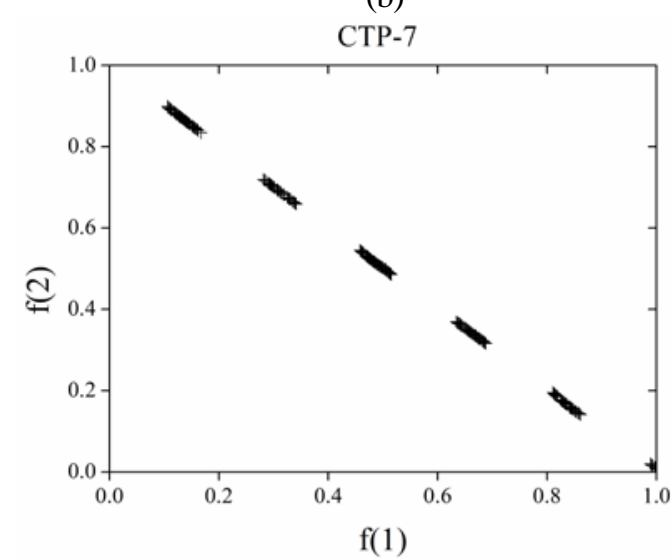

(d)

Fig. 5. Simulation results on the CTP series of benchmark problems 


\section{Simulations}

In this section, we will evaluate our proposed optimization methodology of constellation design in a realistic LEO satellite broadband network scenario. In addition, we also analyze the influence of the main design variables on the performance of satellite networks. In all sImulations, the results are carried out by using both STK (Satellite Tool Kit) and Matlab.

\subsection{Influence Factors Analysis}

According to the aforementioned analysis, optimization variables are important to build a satellite network. In this respect, it is necessary to analyze the influence of the main optimization variables on network coverage and stability.

(1) Network Coverage. We now study the impact of orbital height and inclination on the coverage performance of a single satellite. The orbital height of the satellite is $700 \mathrm{~km}, 1100 \mathrm{~km}$, $1600 \mathrm{~km}$ and $2000 \mathrm{~km}$, respectively. Simulation is done for satellite inclination $i\left(0^{\circ} \leq i \leq 90^{\circ}\right)$ and the user with the minimum elevation angle $E\left(E=10^{\circ}\right)$ in the Northern Hemisphere, as shown in Fig. 6. It is obvious that the satellite with small inclination only covers low latitude area, while the satellite operating at near-polar orbit can cover remote users at all latitude. For covering high latitude area, a satellite with lower orbital height must operate at larger inclination orbit. Hence, it is necessary to take into account both the orbital parameters of the satellite and the location of users in constellation optimization design.

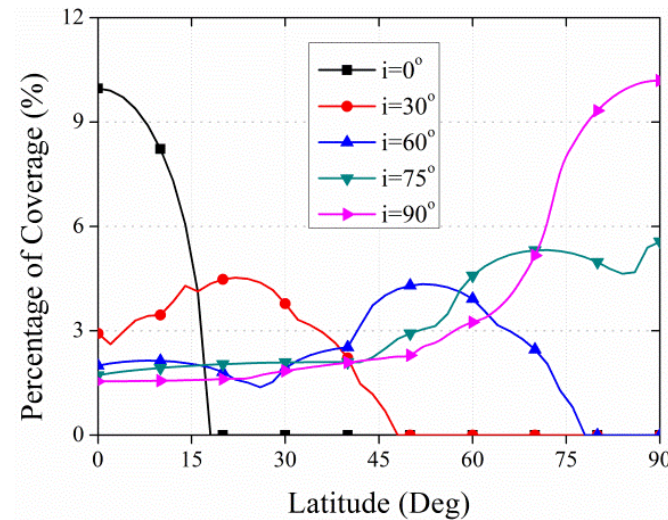

(a) $\mathrm{h}=700 \mathrm{~km}$

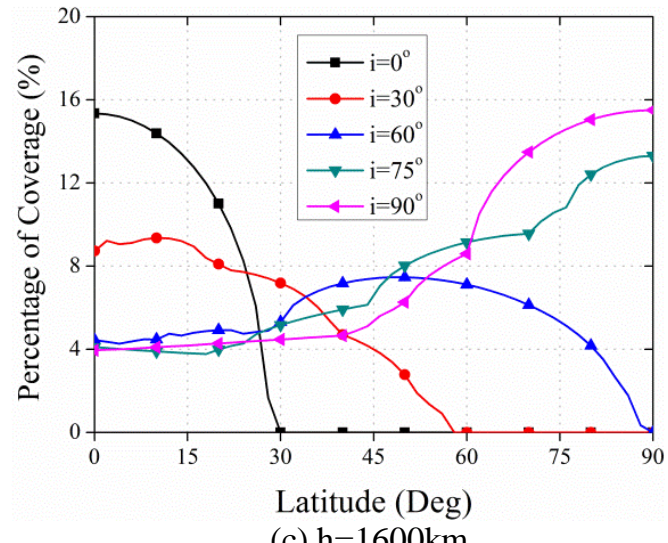

(c) $\mathrm{h}=1600 \mathrm{~km}$

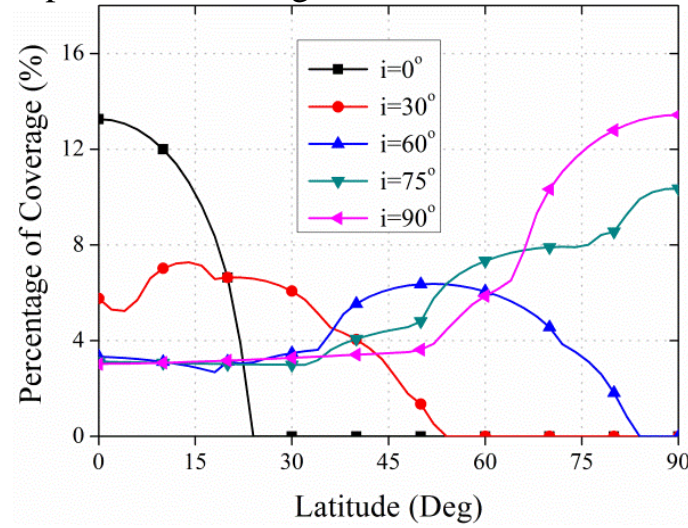

(b) $\mathrm{h}=1100 \mathrm{~km}$

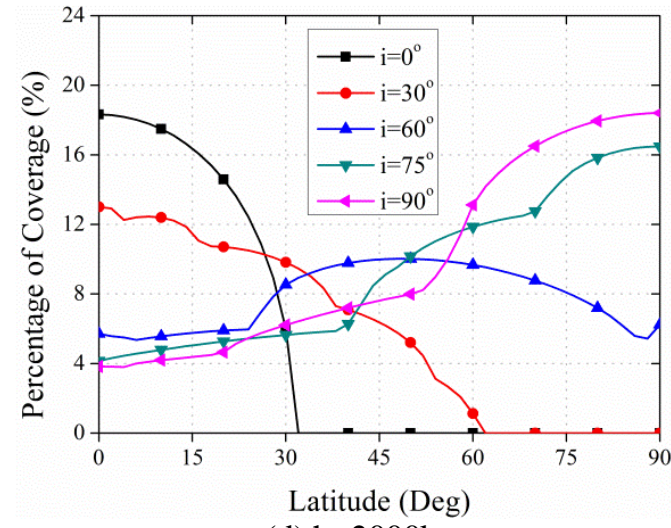

(d) $\mathrm{h}=2000 \mathrm{~km}$

Fig. 6. Percentage of coverage with different satellite inclinations and user latitudes with $E=10$ 


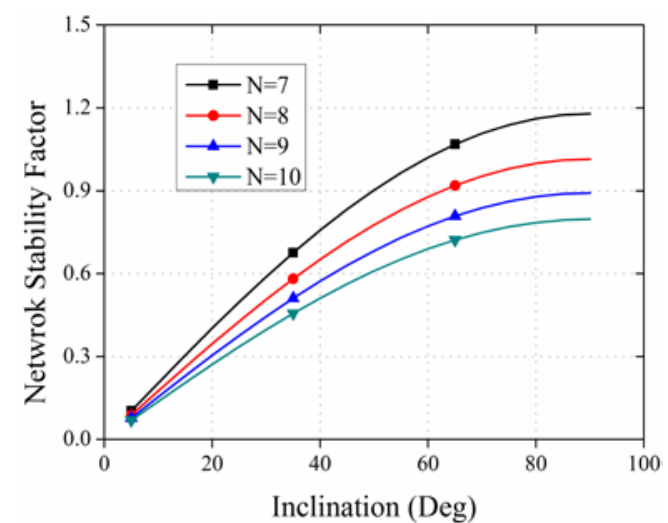

(a) The influence of inclination on network stability

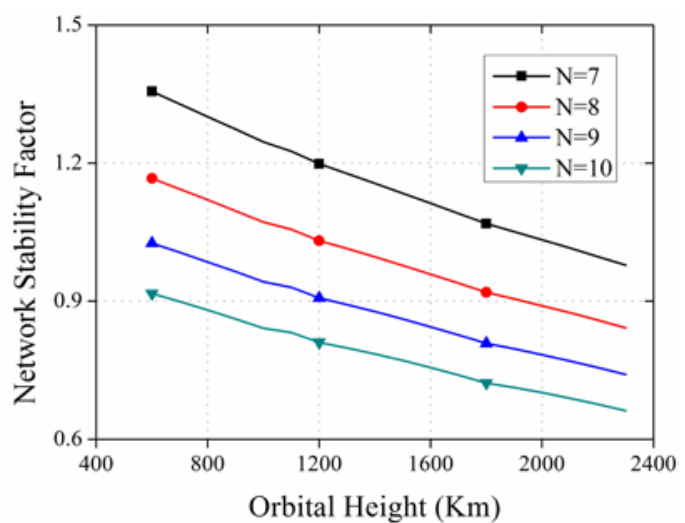

(b) The influence of orbital height on network stability

Fig. 7. The influence of orbit height and inclination on NSF.

(2) Network Stability. In this subsection, we mainly investigate the stability of satellite networks versus the main optimization variables (i.e., orbital height, inclination and the number of orbital planes). Noting that the smaller network stability factor suggests a great opportunity to build the more stable LEO satellite network from section 3.2. Fig. 7 shows the network stability factor for the different number of orbital planes with the various inclination and orbital height, respectively. We can observe from Fig. 7(a) that the stability of the network increases as the number of orbital planes and inclination are decreased. The reason is that the bigger number of orbital planes and the smaller inclination would result in the more dynamic of ISL. From Fig. 7(b), It can be noticed that the network stability factor keeps decreasing as the number of orbital planes and orbital height increase. At the constant number of orbital planes, satellite network will be more stable with the orbital height increasing due to less dynamic ISLs.

These simulation results show that orbital height, inclination and the number of orbital planes have the important influence on the satellite networks. Therefore, we need to make a good trade-off between these parameters in constellation optimization design.

\subsection{Optimization, Analysis, and Comparison of Results}

In order to establish a baseline for all the simulations, the resolution of a grid on the Earth's is spaced $6^{\circ}$ in latitude and longitude respectively and each constellation is evaluated over one day of propagation.

(1) Parameters Setting in Optimization. In order to allow meaningful comparisons, link budget parameters should be kept constant during the whole optimal design process. The values of the link budget parameters are set in Table 3 .

Table 3. Link budget parameters ${ }^{[37]}$

\begin{tabular}{ccc}
\hline Parameter & Symbol & Value \\
\hline Downlink Frequency & $f_{c}$ & $12.2 \mathrm{GHz}$ \\
Required Information Rate & $R_{0}$ & $1.544 \mathrm{Mbps}$ \\
Required Bit Error Rate & $B E R_{0}$ & $10^{-5}$ \\
Required Signal-to-Noise Ratio & $E_{b} / N_{0}$ & $6.4 \mathrm{~dB}$
\end{tabular}




\begin{tabular}{ccc} 
Rain Loss and Link Margin & $L_{R M}$ & $7.8 \mathrm{~dB}$ \\
Multi-Access Efficiency & $\eta_{M A E}$ & $90 \%$ \\
Back-off of Output Power & {$[B O]_{o c}$} & $3 \mathrm{~dB}$ \\
User Terminal System Noise Temperature & $T_{\text {user }}$ & $135 \mathrm{~K}$ \\
User Terminal Aperture Diameter & $D_{\text {user }}$ & $0.6 \mathrm{~m}$ \\
User Terminal Illumination & $\eta_{\text {user }}$ & $60 \%$ \\
\hline
\end{tabular}

In Table 3, the downlink frequency is set as $12.2 \mathrm{GHz}$. It should be noted that the simulations can also be done at the $\mathrm{Ka} / \mathrm{Ku}$ band while the rain loss and link margin need to be modified accordingly. The values of $R_{0}, B E R_{0}$, and $E_{b} / N_{0}$ are the essential requirement for the implementing broadband system. For simplicity, we only consider the multi-access efficiency which is the ratio of usable information rate to the total information rate and ignore the specific multi-access scheme in the simulation. In practice, the satellite transponder must work in a back-off state and $[B O]_{o c}$ is the back-off of output power. The user terminal system noise temperature is assumed to be $135 \mathrm{~K}$. The gain of the user terminal antenna can be obtained by the user terminal aperture diameter and illumination.

The detailed simulation parameters of NSGA- II -IS are listed in Table $\mathbf{4}$ for the proposed constellations optimization design methodology.

Table 4. Parameters of NSGA- II -IS

\begin{tabular}{cc}
\hline Parameters & Values \\
\hline Population Size & 100 \\
Generations & 100 \\
Crossover Probability & 0.9 \\
Mutation Probability & 0.1 \\
Crossover Distribution Index & 15 \\
Mutation Distribution Index & 20 \\
Proportion of Infeasible Solutions & 0.2 \\
\hline
\end{tabular}

(2) Baseline Optimization Results. Fig. 8 depicts the non-dominated solutions generated by NSGA- II -IS during the run of the constellation design problem.

Studying the results in Fig. 8 provides powerful insight into the tradeoff between cost-efficient and network stability. It is obvious that network capacity per cost of the solutions rises as the network stability factor increases. This indicates that cost-efficient of the network will improve with the decrease of network stability. Further conclusions can be drawn from Fig. 8. The approximate Pareto fronts suggest generally improving cost-efficient of the network and reducing the network stability for decreased the number of orbital planes. Fig. 8 also illustrates that with fixing the number of orbital planes, cost-efficient of the network degrades as the total number of satellites increase. 


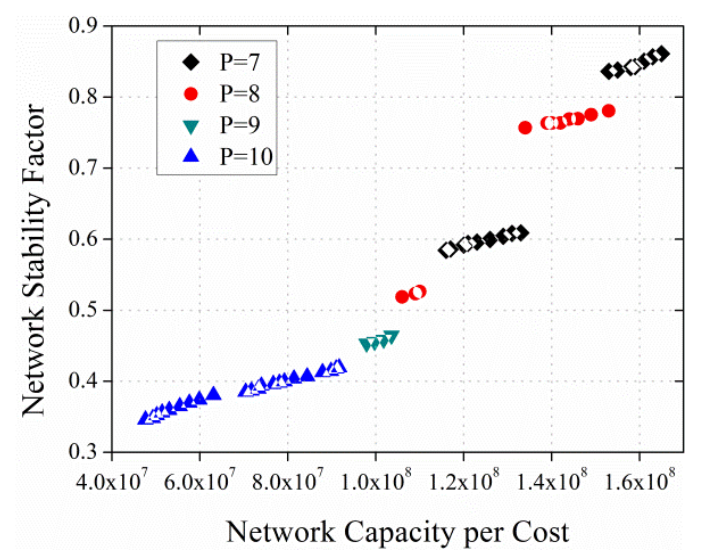

Fig. 8. Tradeoff between NCPC and NSF

As shown in Fig. 8, there exists a 'steep' characteristic of the approximate Pareto fronts. The reason for such a situation is that the change of the total number of satellites is discontinuous in the optimization process.

(3) Optimization Variable Analysis. Each data point made up of the non-dominated fronts in Fig. 8 represents a set of optimization variables that define a constellation for a satellite network. To better understand the designs discovered by the optimization algorithm, orbital height and inclination are respectively plotted versus the two optimization objective for all Pareto solutions.

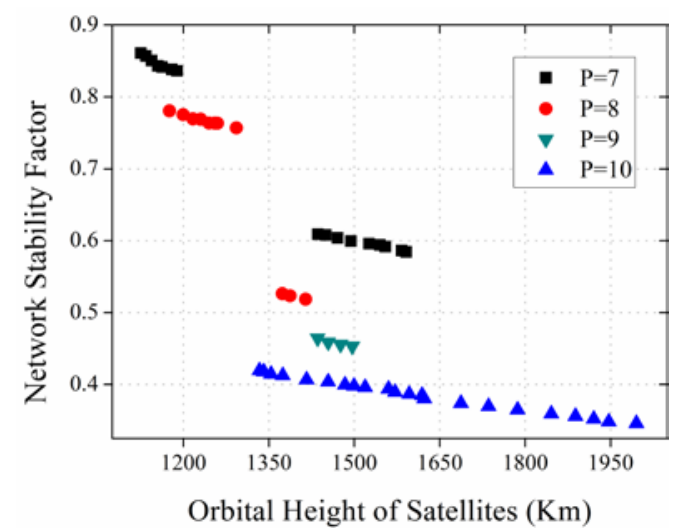

Fig. 9(a). Influence of orbital height on NSF

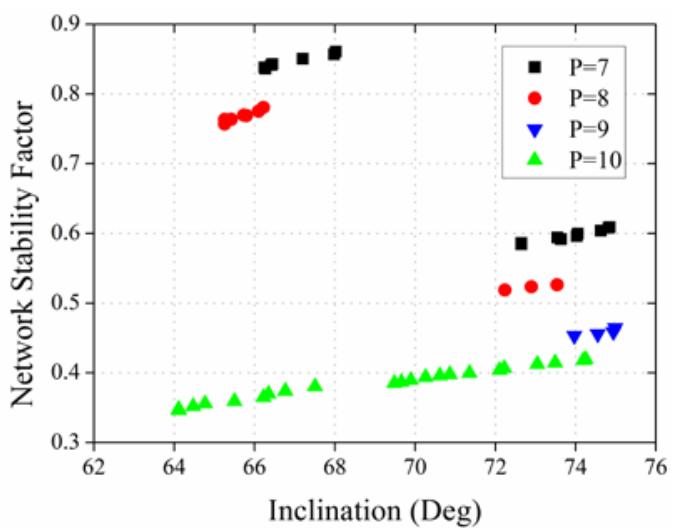

Fig. 9(b). Influence of inclination on NSF

The solutions in Fig. 9 are orbital height and inclination versus network stability, respectively. At the constant number of orbital planes, the stability of the network is enhanced with the increase of orbital height, and the network stability is degraded due to the more frequent changes in ISLs as the inclination increases. The optimization results and the simulation results in section 6.1 match well. However, as the number of orbital plane decreases, the network is less stable when orbital height and inclination of the constellation is fixed.

Fig. 10 reflect the influence of the orbital height and inclination on the network capacity per cost. In order to realize a higher network cost-efficient ratio, orbital height should be lower and the inclination should be less with fixing the number of orbital planes. As the number of orbital 
plane increases, the network capacity per cost will decrease when the orbital height and inclination remain constant.

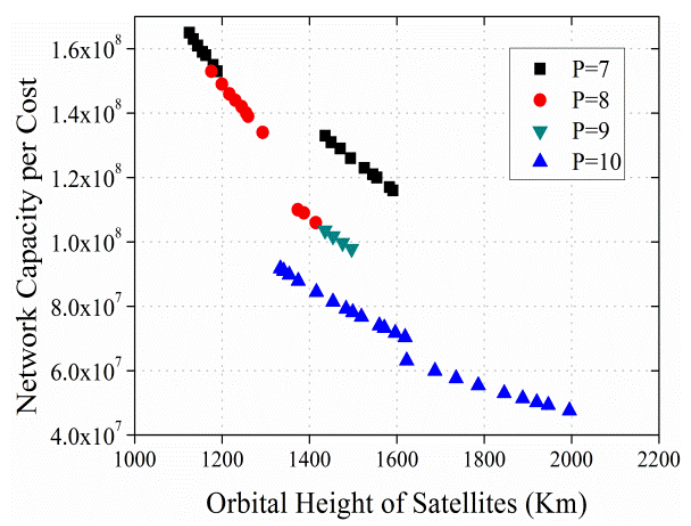

Fig. 10(a). Influence of orbital height on NCPC

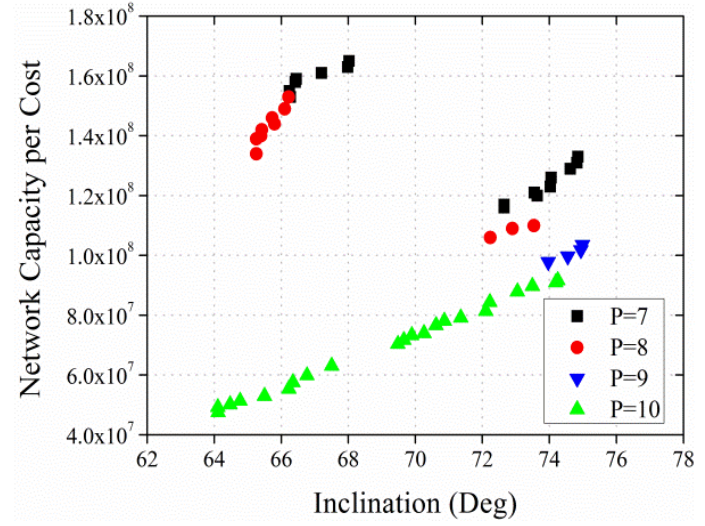

Fig. 10(b). Influence of inclination on NCPC

Moreover, it can be summarized that the proposed algorithm could maintain the diversities of the solution spaces from these simulation results. It also means that constellations with lower orbital height and inclination are preferred for LEO satellite network.

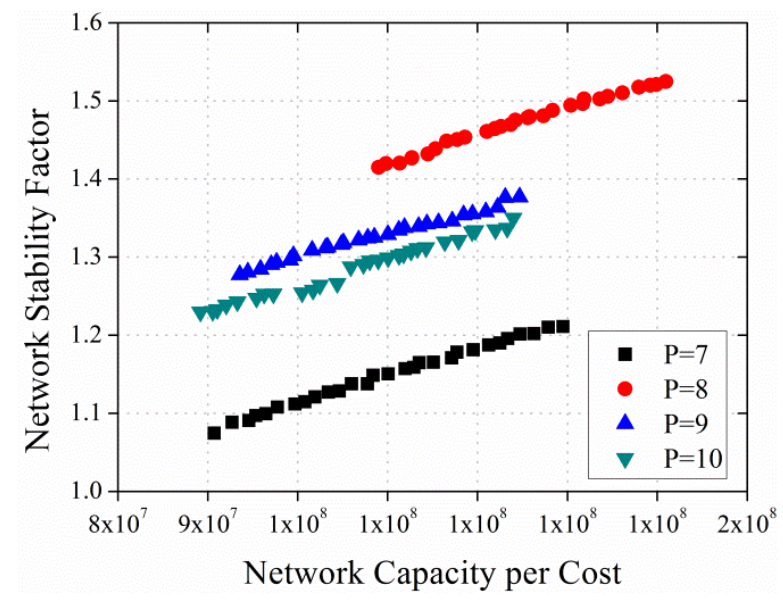

Fig. 11. Pareto fronts for seven-, eight-, nine-,and ten-orbital planes of constellations

(4) Performance Comparison. The Pareto fronts found by the optimization algorithm for seven-, eight-, nine-, and ten-orbital planes of constellations are shown in Fig.11 when the other optimization variables and parameters maintain consistently in each optimization process. These results further show the tradeoff between between cost-efficient and network stability.

A comparison of the constellations for LEO satellite broadband networks with the same cost-efficiency is carried out in this section. The motivation is to discover how to choose an optimal constellation in practical application. At the constant network capacity per cost of $1.17 \times 10^{8}$, the values of optimization variables are shown in Table 5. Fig 12 compares the envisaged four different LEO satellite networks in terms of some important parameters, such as orbital height, inclination, system complexity (i.e. the total number satellites in the 
constellation), satellite payloads (i.e. the equivalent area of satellite downlink antenna and transmission power of a satellite), and network capacity.

Table 5. Comparison of constellation designs with the same cost-efficient

\begin{tabular}{ccccccc}
\hline $\mathbf{( T / P / F )}$ & $\boldsymbol{h} / \mathbf{k m}$ & $\mathbf{i} / \mathbf{D e g}$ & $\boldsymbol{A}_{\text {sat }} \boldsymbol{m}^{2}$ & $\boldsymbol{P}_{\text {sat }} / \boldsymbol{w}$ & $\boldsymbol{N S F}$ & Capacity \\
\hline $\mathbf{5 6 / 7 / 0}$ & 1571.7 & 64.2 & 1.43 & 414.4 & 1.18 & $3.88 * 10^{12}$ \\
$\mathbf{6 4 / 8} / \mathbf{1}$ & 1425.0 & 63.7 & 1.24 & 351.3 & 1.45 & $3.26 * 10^{12}$ \\
$\mathbf{9 9 / 9 / 1}$ & 1039.6 & 67.8 & 0.91 & 283.0 & 1.35 & $2.98 * 10^{12}$ \\
$\mathbf{9 0 / 1 0 / 1}$ & 1109.4 & 67.1 & 1.19 & 219.1 & 1.32 & $2.75 * 10^{12}$ \\
\hline
\end{tabular}

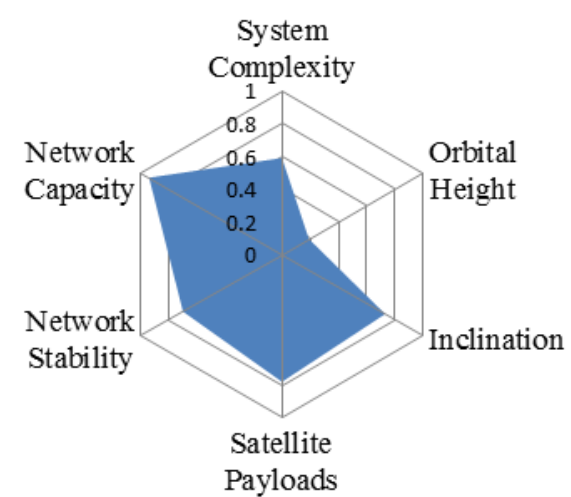

(a) 56/7/0 constellation

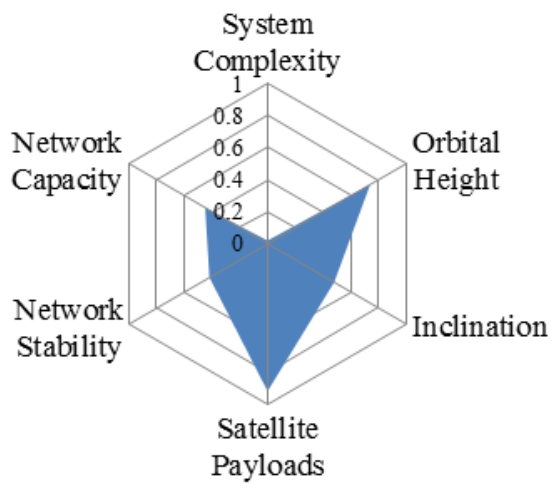

(c) 99/9/1 constellation

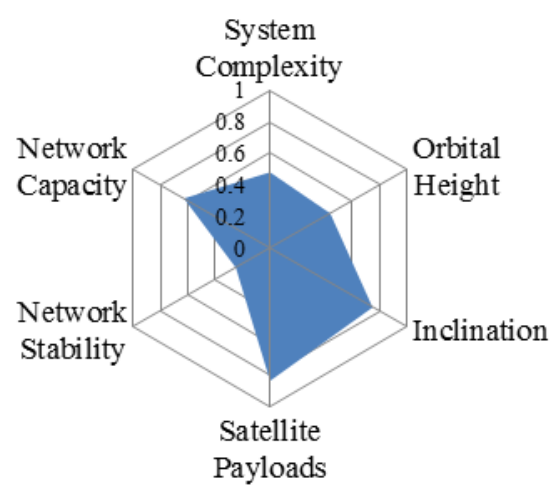

(b) 64/8/1 constellation

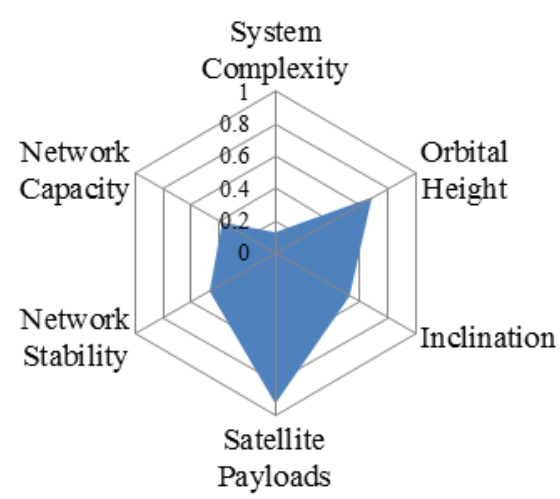

(d) 90/10/1 constellation

Fig. 12. Comparison of different LEO satellite broadband network

The parameter values used for obtaining Fig. 12 are normalization of the data from Table 5. It can be seen that the greater the shadowed area is for one system in Fig. 12, the better its overall evaluation is. Hence, the constellation with seven orbital planes appears to achieve the best performance as the architecture of the LEO satellite broadband network.

Fig. 13 provides the coverage performance of the constellation (56/7/0) reported by STK. In this paper, the coverage multiplicity is used to estimate the coverage performance of the constellation. Coverage multiplicity is the number of satellites in sight of the users in each grid. From Fig. 13, the contour shows that global seamless coverage can be achieved by the constellation (56/7/0). More than $97 \%$ of the earth surface can be covered by at least two satellites. The region between north latitude $30^{\circ}-70^{\circ}$, where people act multifariously and 
concentratedly, can be covered by three or more satellites. Even in polar regions, there are at least one satellites in sight of the user terminals.

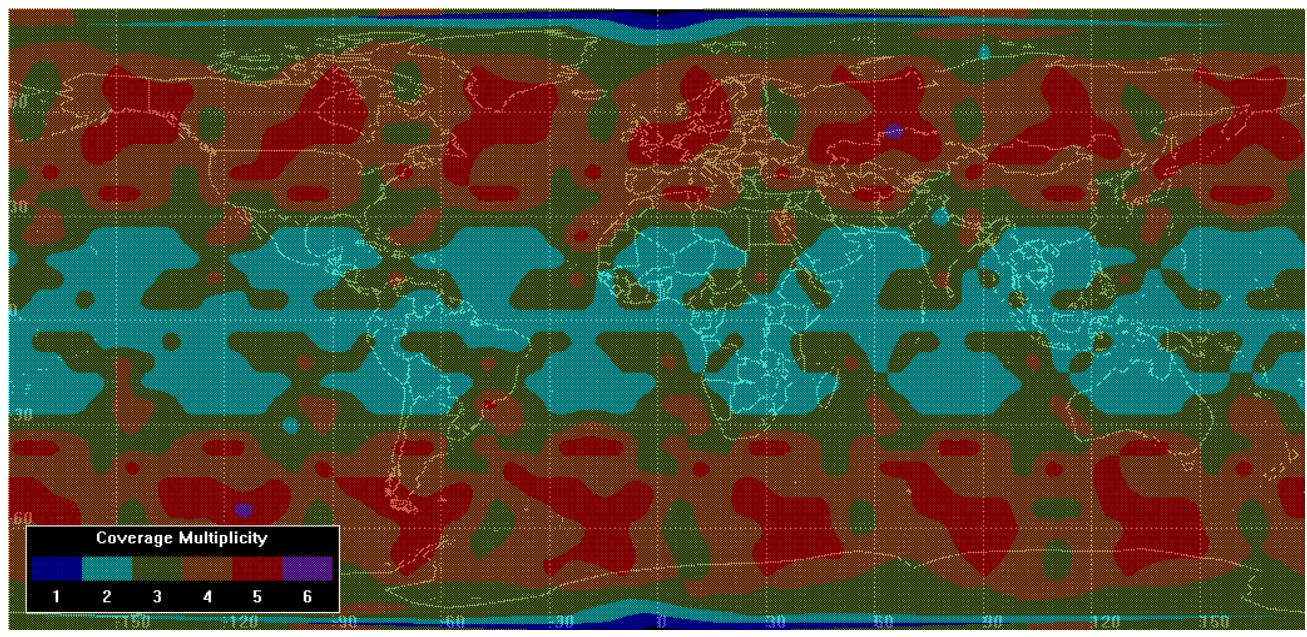

Fig. 13. Coverage multiplicity of the constellation (56/7/0)

Fig. 14 shows the average elevation of user terminals in the satellite network supported by the constellation (56/7/0). The satellite network can provide a better broadband communication quality for user terminals located between about $70^{\circ} \mathrm{N}$ and $70^{\circ} \mathrm{S}$ because the average elevation of them is more than $25^{\circ}$. Even in polar regions, user terminals can get effective communication service due to the average elevation larger than $10^{\circ}$.

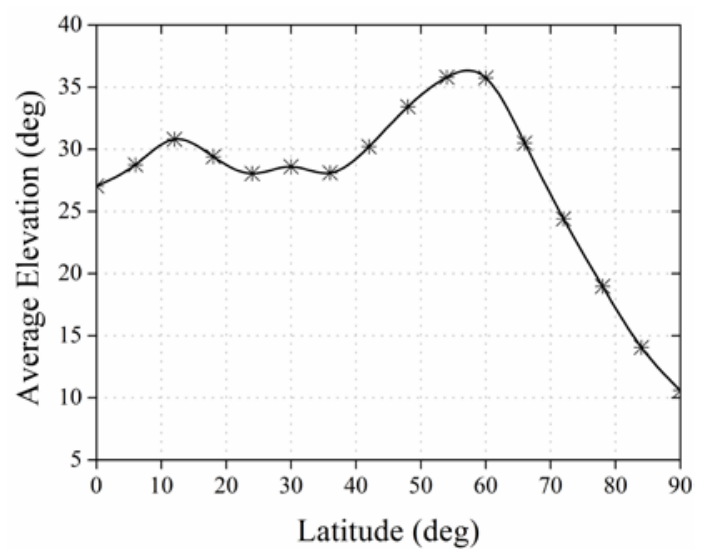

Fig. 14. Average elevation of user terminals compare to different latitudes

\section{Conclusions}

This paper studied optimization methodology of constellation design in LEO satellite broadband network. The optimization model of constellation design takes comprehensive factors into account, including QoS metrics, network stability, network capacity, and network construction cost. In addition, improved NSGA- II with constraints handling is proposed to solve the optimization model. The Pareto fronts provide more choice for the decision maker to 
design a suitable satellite constellation with a trade-off in the network cost-efficiency and stability. The simulation results validate that the proposed optimization methodology of constellation design can guide the optimal deployment of the LEO satellite network as well as SIN planning and construction.

In the future work, we plan to relax the assumptions as stated in the previous section and make the optimization model close to reality. The practicality of designed constellation will be greatly improved in actual application. Moreover, to further improve the performance of the satellite network, it requires research on the constellation optimization design for multi-layer satellite networks.

\section{References}

[1] C. Jiang, X. Wang, J. Wang, H. Chen, and Y. Ren, “Security in Space Information Network,” IEEE Communications Magazine, no. 8, pp. 82-88, August, 2015. Article (CrossRef Link)

[2] J. Du, C. Jiang, Q. Guo, M. Guizani, and Y. Ren, "Cooperative earth observation through complex space information networks," IEEE Wireless Communications, no. 4, pp.136-144, May, 2016. Article (CrossRef Link)

[3] P. Chini, G. Giambene, and S. Kota, "A survey on mobile satellite systems," International Journal of Satellite Communications, vol. 28, no. 1, pp. 29-57, August, 2010. Article (CrossRef Link)

[4] D. Vallado, Fundamentals of astrodynamics and applications, Space Technology Library, Microcosm Press, California, USA, 2007.

[5] J. Wang, L. Li, and M. Zhou, "Topological dynamics characterization for LEO satellite networks," Computer Networks, vol. 51, no. 1, pp. 43-53, January, 2007. Article (CrossRef Link)

[6] D. Mortari, M. D. Sanctis, and M. Lucente, "Design of Flower Constellations for telecommunication services," Proceedings of the IEEE, vol.99, no. 11, pp. 2008-2019, November, 2011. Article (CrossRef Link)

[7] J. G. Walker, "Some circular orbit patterns providing continuous whole earth coverage," Journal of British Interplanetary, vol.24, no. 1, pp. 369-384, 1971.

[8] D. Mortari, M. P. Wilkins, and C. Bruccoleri, "The flower constellations," Journal of Astronautical Sciences, vol. 52, no.1, pp. 107-127, 2004.

[9] H. Keller, H. Salzwedel, and G.Schorcht, "Geometric aspects of polar and near polar circular orbits for the use of inter-satellite links for global communication," in Proc. of 48th IEEE Vehicular Technology Conference, pp. 199-203, May 21, 1998. Article (CrossRef Link)

[10] Y. Ulybyshev, "Geometric analysis and design method for discontinuous coverage satellite constellations," Journal of Guidance, Control, and Dynamics, vol.37, no.2, pp. 549-557, February, 2012. Article (CrossRef Link)

[11] E. A. Williams, W.A. Crossley, and T. J. Lang, "Average and maximum revisit time trade studies for satellite constellations using a multi-objective genetic algorithm," The Journal of Astronautical Sciences, vol. 49, no. 3, pp. 385-400, 2000.

[12] W. Mason, C. Victoria, and J. Hartmann, "Optimal earth orbiting satellite constellations via a Pareto Genetic Algorithm," AIAA/AAS Astrodynamics Specialist Conference and Exhibit, pp. 169-174, August 10-12, 1998. Article (CrossRef Link)

[13] M. P. Ferringer, R. S. Clifton, and T. G. Thompson, "Efficient and accurate Evolutionary Multi-Objective Optimization paradigms for satellite constellation design," Journal of Spacecraft and Rockets, vol. 44, no. 3, pp. 682-691, May, 2007. Article (CrossRef Link)

[14] M. P. Ferringer, and D. B. Spencer, "Satellite constellation design tradeoffs using Multiple-Objective Evolutionary Computation,” Journal of Spacecraft and Rockets, vol. 43, no. 6, pp.1404-1411, November, 2006. Article (CrossRef Link)

[15] W. R. Whittecar, and M. P. Ferringer, "Global coverage constellation design exploration using Evolutionary Algorithms," AIAA/AAS Astrodynamics Specialist Conference, pp. 4159-4163, San.Diego, CA, August 4-7, 2014. Article (CrossRef Link) 
[16] A. L. Jennings, and H. Diniz, "Global navigation satellite system design exploration using a Multi-Objective Genetic Algorithm,” in Proc. of AIAA Space 2015 Conference and Exposition, pp. 4622-4631, Pasadena, California, USA, August 1-3, 2015. Article (CrossRef Link)

[17] M. Curry, L. T. Paul, and S. Slagowski, "Multidisciplinary design optimization for a high-resolution Earth-imaging constellation,” in Proc. of 2015 IEEE Aerospace Conference, pp. 1-10, March 7-4, 2015. Article (CrossRef Link)

[18] J. Yong, S. Feng, Z. Xie. G. Zhang, and G. Li, “A novel MEO constellation for global communication without Inter-Satellite Links,” in Proc. of 2010 IEEE Int. Conf. on Wireless Communications and Signal Processing (WCSP), pp.1-5, October 21-23, 2010. Article (CrossRef Link)

[19] Miller, "Multi-Objective, multidisciplinary design optimization methodology for distributed satellite systems," Journal of Spacecraft and Rockets, vol. 41, no. 1, pp. 39-50, January, 2004. Article (CrossRef Link)

[20] K. Deb, S. Agrawal, A. Pratap, and T. Meyarivan, “A fast elitist non-dominated sorting genetic algorithm for multiobjective optimization: NSGA- II ,” in Proc. of 6th Int. Conf. on Parallel Problem Solving From Nature, pp. 849-858, September 18 - 20, 2000.

[21] H. Xu, and X. Zhou, "Ka-band satellite channel model in deep space relaying network," Wireless Personal Communications, vol.77, no.2, pp.881-888, July, 2014. Article (CrossRef Link)

[22] J. Restrepo, G. Maral, G. Massy, and J. Velez, "Evaluation of traffic performances in non-Geo satellite constellation for mixed services (mobile and fixed)," in Proc. of 20th AIAA International Communication Satellite Systems Conference and Exhibit, pp. 1-8, May 12-15, 2002. Article (CrossRef Link)

[23] Z. Wang, P. Wang, X. Gu, and Q. Guo, "Research on design of permanent inter-satellite-links in satellite networks,” Journal on Communications in China, vol.27, no. 8, pp.129-133, August, 2006.

[24] J. Wang, Y. Hu, H. Zhou, and M. Zhou, “Topological dynamics characterization for layered satellite networks,” Computer Networks, vol. 51, no. 1, pp. 43-53, January, 2007. Article (CrossRef Link)

[25] J. R. Wertz, and W. J. Larson, Space misson analysis and design, Kluwer Academic Publishers. Dordrecht, The Netherlands. 1991.

[26] N. Kumar, and J. H. Lee, “Collaborative-Learning-Automata-based channel assignment with topology preservation for wireless mesh networks under QoS constraints,” IEEE Systems Journal, vol. 9, no. 3, pp. 675-685, September, 2015. Article (CrossRef Link)

[27] H. Xu, and X. Zhou, "A non-cooperative differential game model for frequency reuse based channel allocation in satellite networks," Wireless Personal Communications, vol.79, no.1, pp.405-416, November, 2014. Article (CrossRef Link)

[28] F. Dong, H. Han, J. Wang, and H. Li, “A constellation design methodology based on QoS and user demand in High Altitude Platform broadband networks,” IEEE Transactions on Multimedia, vol. 18, no. 12, pp. 2384-2397, December, 2015. Article (CrossRef Link)

[29] P. Gvozdjak, Modeling communications in low-earth-orbit satellite networks[Doctor Thesis], Simon Fraser University, Vancouver, Canada. 2000.

[30] B. Gavish, and J. Kalvenes, "The impact of intersatellite communication links on LEOS performance,” Telecommunication Systems, vol. 8, no. 2, pp. 159-190, December, 1997. Article (CrossRef Link)

[31] J. Zhang, Space-based mobile communication network, Beijing, China, National Defense Industry Press, 2011.

[32] E. Mezura-Montes, and C. A. C. Coello, "Constraint-handling in nature-inspired numerical optimization: Past, present and future,” Swarm and Evolutionary Computation, vol. 1, no. 4, pp. 173-194, December, 2011. Article (CrossRef Link)

[33] Z. Michalewicz, and M. Schoenauer, "Evolutionary algorithms for constrained parameter optimization problems," Evolutionary computation, vol. 4, no. 1, pp. 1-32, December, 1996. Article (CrossRef Link) 
[34] C. A. C. Coello, "Theoretical and numerical constraint-handling techniques used with evolutionary algorithms: a survey of the state of the art." Computer Methods in Applied Mechanics and Engineering, vol. 191, no. 11, pp. 1245-1287, January, 2002. Article (CrossRef Link)

[35] J. B. Kollat, and P. M. Reed, "The Value of Online Adaptive Search: a Performance Comparison of NSGA-II, $\varepsilon$-NSGA-II , and عMOEA," in Proc. of Int. Conf. on Evolutionary Multi-Criterion Optimization, pp. 386-398, March 9-11, 2005. Article (CrossRef Link)

[36] Y. G. Woldesenbet, G. G. Yen, and B. G. Tessenma, "Constraint Handling in Multi-objective Evolutionary Optimization," in Proc. of 2007 IEEE Congress on Evolutionary Computation, pp. 514-525, September 25-28, 2009. Article (CrossRef Link)

[37] T. Kashitani, Development and application of an analysis methodology for satellite broadband network architectures[Doctor Thesis], Massachusetts Institute of Technology, Cambridge, USA, 2002.

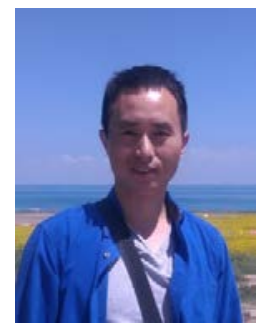

Dawei Yan was born in Shaanxi Province, China, in 1986. He received the B.S. from the College of Aeronautics and Astronautics Engineering, Air Force Engineering University, Xi'an, China, in 2010. He is currently working toward the $\mathrm{PhD}$ degree in electrical engineering at the College of Electronic Science, National University of Defense Technology, Changsha, China. His current research interests include space information network, satellite communication.

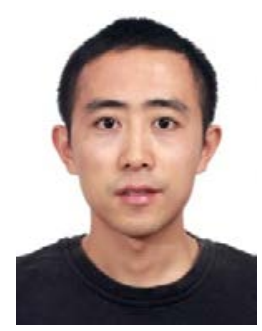

Peng You was born in Jiangxi Province, China. He received the B.S., M.S., and Ph.D. degrees all in electrical engineering from the National University of Defense Technology (NUDT), Changsha, China, in 2005, 2008, 2014. Since 2014, he has been a Lecturer with the School of Electronic Science and Engineering, NUDT. His current research interests include the design of architecture for space information network, cognitive radar. 


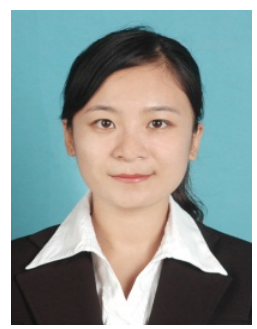

Cong Liu was born in Shaanxi Province, China, in 1987. He received the B.S. degrees from Zhengzhou University, Zhengzhou, China, in 2011. He is now a lecturer in the College of Mathematics and Information Science, Baoji University of Arts and Sciences, Baoji, China. Her current research focuses on algorithm design.

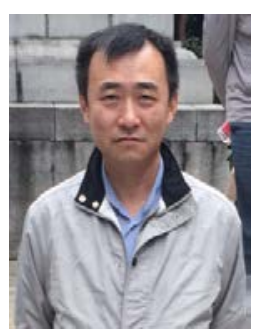

Shaowei Yong was born in Chongqing, China, in 1965. He received the B.S. degree from the College of Communications Engineering, Nanjing, China, in 1986 and the M.S., Ph.D. degrees from the National University of Defense Technology (NUDT), Changsha, China, in 1989, 1997, all in electrical engineering. He is now a professor in the College of Electronic Science and Engineering, National University of Defense Technology, Changsha, China. His current research interests include space information network, satellite communication.

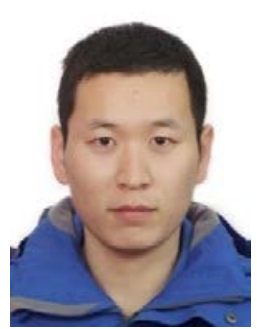

Dongfang Guan was born in Henan Province, China, in 1988. He received the B.S. and Ph.D. degrees from the College of Communications Engineering, PLA University of Science and Technology, Nanjing, China, in 2011 and 2016. He is now a lecturer in the College of Electronic Science, National University of Defense Technology, Changsha, China. His current research interests include microstrip antennas, array antenna, SIW technology, spoof surface plasmons and metamaterials. 